\title{
Microgastria congénita, hallazgos radiológicos. Presentación de caso y revisión de la literatura
}

\section{Congenital microgastria, radiological findings. Case report and}

\section{literature review}

María T. Silva-Martínez ${ }^{1 *}$, Sara A. Solórzano-Morales ${ }^{1}$, Edgar I. Torres-Estrada ${ }^{2}$ y

Leslie R. Villanueva-Bárcenas ${ }^{3}$

${ }^{1}$ Departamento de Radiología, Instituto Nacional de Pediatría; ${ }^{2}$ Departamento de Radiología e Imagen, Hospital de Especialidades Belisario Domínguez;

${ }^{3}$ Departamento de Pediatría, Instituto Nacional de Pediatría. Ciudad de México, México

\section{RESUMEN}

La microgastria congénita es una anomalía extremadamente rara en la que el desarrollo embrionario del intestino anterior se detiene, dando lugar a un estómago pequeño, de forma tubular o sacular, por lo general asociado a un megaesófago. Se describe el caso de una niña con microgastria congénita y se discuten la embriología, la presentación clínica, las anomalías asociadas, los métodos de imagen (serie esofagogastroduodenal y ultrasonido abdominal) y el tratamiento quirúrgico.

Palabras clave: Microgastria congénita. Malformaciones gástricas. Megaesófago. Reflujo gastroesofágico. Gastroyeyunostomía.

\section{ABSTRACT}

Congenital microgastria is an extremely rare disease in which the anterior bowel stops, giving way to a small stomach, tubular or sacular in shape, generally associated to megaesophagus. In this paper we describe the case of a girl with congenital microgastria and we discuss the embryology, clinical presentation, associated anomalies, imaging methods such as esophageal and gastroduodenal study and abdominal sonogram, and surgical treatment.

Key words: Congenital microgastria. Gastric malformations. Megaesophagus. Gastroesophageal reflux. Gastrojejunostomy. 


\section{INTRODUCCIÓN}

Comparadas con las malformaciones de otras regiones del tracto gastrointestinal, las anomalías congénitas del estómago son infrecuentes. Los divertículos del estómago, las duplicaciones gástricas y los defectos musculares congénitos son $\operatorname{raros}^{1-11}$; la aplasia del estómago o agastria y la microgastria son condiciones sumamente raras $^{2}$. La microgastria congénita se reportó por primera vez en 1894, por Dide ${ }^{3,8,10,11}$, pero hasta 1971 Schultz y Niemann la describieron como una condición clínica ${ }^{1,8,10}$. El estómago se origina como una dilatación fusiforme del intestino anterior ${ }^{3-5,7,11,12}$ entre la cuarta y la quinta semana de gestación ${ }^{3,4,7,10-12}$; si su desarrollo se detiene, se produce microgastria como anomalía aislada ${ }^{9,11} \mathrm{o}$, más frecuentemente, en conjunto con otras malformaciones del intestino anterior, medio y posterior, como la asociación VACTERL, que incluye anomalías vertebrales, ano imperforado, atresia de esófago, fístula traqueoesofágica, anomalías renales y de extremidades ${ }^{3-9}$, atresia duodenal ${ }^{5,9}$, asplenia ${ }^{2-9}$, malrotación intestinal ${ }^{1,3,5-8}$, anomalías cardiopulmonares $3,5,6,7,9$ y del sistema nervioso central, hendiduras laringotraqueales ${ }^{6}$, enfermedad de Hirschprung 5,9, hernia diafragmática ${ }^{4}$, estenosis del esófago distal $^{4}$ y ausencia de vesícula biliar ${ }^{8}$.

Las manifestaciones clínicas varían de un paciente a otro, dependiendo de la etapa en la que el desarrollo del estómago se detiene. La presentación prenatal es parecida a la atresia de esófago, y la falta de visualización del estómago en el segundo trimestre del embarazo sugiere la posibilidad de atresia de esófago o microgastria ${ }^{3}$. Después de nacer, los pacientes con microgastria presentan vómitos posprandiales frecuentes, reflujo gastroesofágico (RGE), neumonías por aspiración recurrentes y desnutrición ${ }^{3-10}$.

El diagnóstico de microgastria congénita se puede realizar con un estudio contrastado del tracto gastrointestinal superior ${ }^{3,5}$, el cual muestra un estómago en la línea media ${ }^{3}$ pequeño, sin fondo, cuerpo ni antro ${ }^{4,5,9}$, de forma tubular o sacular ${ }^{1,5,9,11}$, y frecuentemente con incompetencia del esfínter esofágico inferior ${ }^{1,2,4,6-9}$; también se observa que el esófago se dilata para compensar la incapacidad del estómago de mantener su contenido, y la microgastria casi siempre se acompaña de RGE y de megaesófago ${ }^{1,3-5,11}$. Las biopsias de estómago en pacientes con microgastria muestran una mucosa gástrica normal. A pesar de esta diferenciación celular normal, la masa celular total del estómago está muy reducida y hay escasez de células parietales, probablemente por hipoplasia de todo el estómago, lo que lleva a una disminución de la producción de ácido y de factor intrínseco ${ }^{5}$.

Aunque la etiología de la microgastria sigue siendo desconocida, los estudios de biología molecular y de genética pueden explicar posibles mecanismos moleculares. La asociación no aleatoria de la microgastria con anomalías cardiacas, traqueoesofágicas y renales, anales, vertebrales y de extremidades, sugiere la posibilidad de un defecto común en el desarrollo temprano del mesodermo ${ }^{5,12}$.

\section{CASO CLÍNICO}

Niña nacida el 27 de julio de 2017 de padres originarios de Guerrero, que niegan consanguinidad o endogamia; tiene una hermana 
sana. Producto de la gesta II, embarazo aparentemente normoevolutivo, la madre acudió a siete controles prenatales y seis ecografías con reporte normal. A la madre se le diagnosticó colelitiasis al segundo mes de gestación y pancreatitis aguda al octavo mes, decidiéndose la interrupción del embarazo (por cesárea) a las 34 semanas de gestación, obteniendo un producto único con peso de $2 \mathrm{~kg}$ y talla de $43 \mathrm{~cm}$, que requirió maniobras de reanimación avanzada e intubación endotraqueal con apoyo de ventilación mecánica por 3 días y campana cefálica por 1 semana; en total, permaneció hospitalizada 1 mes por presentar enterocolitis necrosante, manteniéndose en ayuno y aparentemente asintomática. Es dada de alta e inicia con vómitos posprandiales con cada toma del seno materno, referidos como explosivos, aproximadamente ocho al día. Fue valorada por facultativo, quien diagnostica RGE e indica tratamiento antirreflujo y cambio a fórmula, sin mejoría, por lo que acude a otro médico, quien le indica realizar una serie esófago gastro duodenal (SEGD) el día 5 de octubre (70 días de vida extrauterina) en su entidad de origen, reportando dilatación esofágica, y es referida a nuestra institución.

A su ingreso se sospecha estenosis hipertrófica de píloro. El día 12 de octubre (77 días de vida extrauterina) se le realiza endoscopía, en la que se encuentran dilatación esofágica y estómago pequeño.

Se realiza SEGD con opacificación del esófago y dilatación gradual (Fig. 1 A), con aumento en sus movimientos peristálticos (Fig. 1 B), sin vaciamiento inicial, con apertura del hiato esofágico persistentemente, sin observar la unión esofagogástrica y en continuidad con el estómago redondo, medial y pequeño (Fig. $1 \mathrm{C}$ ), con regreso de bario al esófago y volviéndolo a ver vacío (Fig. 1 D). Una vez distendido el estómago, su vaciamiento es lento (Fig. 2 A), pero permitiendo la opacificación del píloro, el bulbo y la arcada duodenal (Fig. 2 B).

También se le realiza ecografía abdominal, que descarta hipertrofia de píloro, pero se observa RGE grave y continuo (Fig. 3 A), y esófago dilatado por el contenido gástrico, con diámetros de hasta 15 mm (Fig. 3 B); el estómago tuvo un volumen de 10 cc (Fig. 3 C y D).

Además, se realiza exploración de colon con enema y se observan microcolon y malrotación (Fig. 4).

Se decide realizar un procedimiento de Hunt Lawrence y el día 8 de noviembre (104 días de vida extrauterina) es sometida a gastroyeyunoanastomosis con «Y» de Roux (asa yeyunal con reservorio en «J») (Fig. 5).

\section{DISCUSIÓN}

La microgastria congénita es una anomalía extremadamente rara del estómago que resulta de la detención del desarrollo normal del intestino anterior. En condiciones normales, el estómago se origina como una dilatación del intestino anterior en la cuarta semana de desarrollo embriológico. En la quinta semana, el estómago modifica su forma y situación por diferencias en la rapidez de crecimiento de diversas porciones de la pared y de cambios en la posición de los órganos adyacentes. Esto explica perfectamente los hallazgos encontrados en la SEGD: estómago pequeño, 

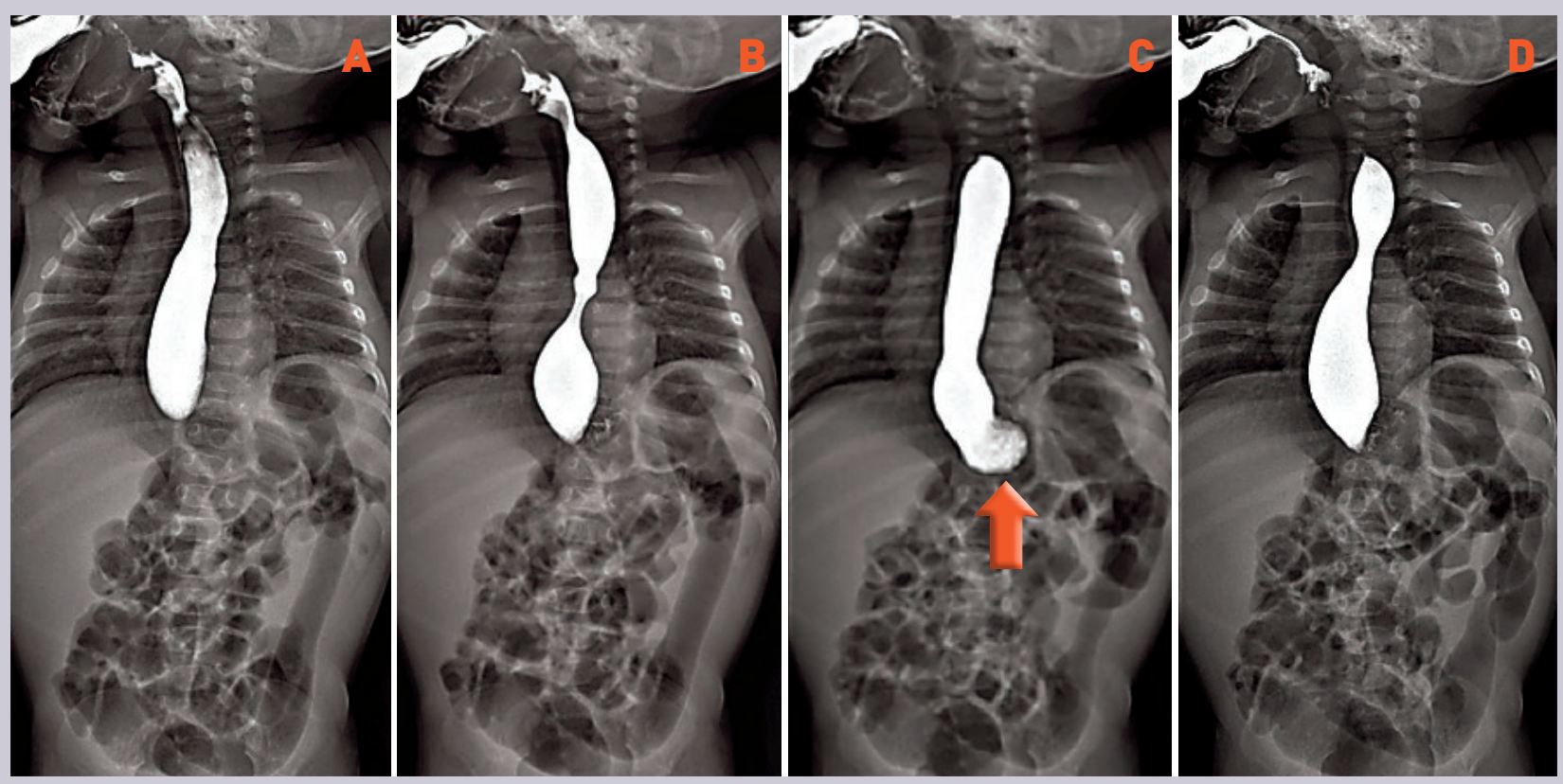

Figura 1. A: esófago sin vaciamiento y con dilatación gradual. B: esófago con aumento en sus movimientos peristálticos. C: apertura del hiato esofágico en continuidad con el estómago redondo, medial y pequeño (flecha). D: el estómago regresó su contenido hacia el esófago y está vacío.
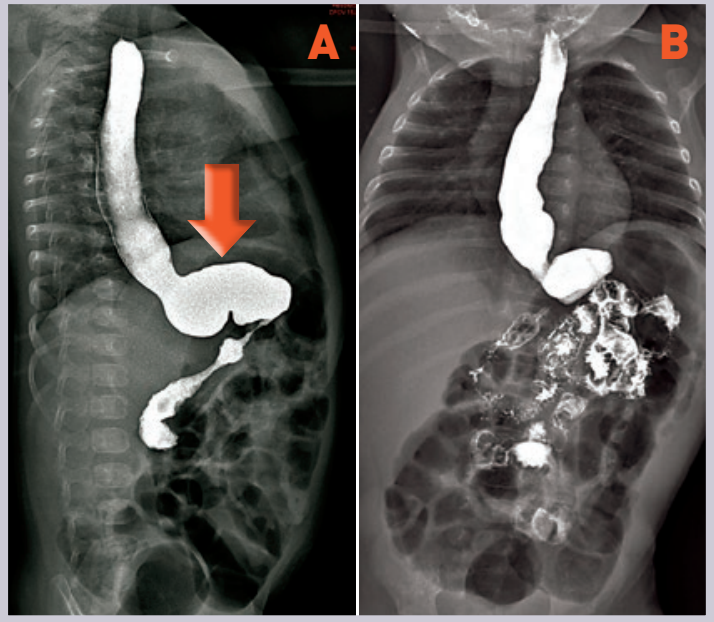

Figura 2. A: el vaciamiento del estómago (flecha) es lento, pero permite la opacificación del píloro, el bulbo y la arcada duodenal. B: vaciamiento parcial del estómago hacia el esófago.

de forma tubular o sacular, sin fondo, cuerpo ni antro, y en la línea media. Durante su desarrollo, el estómago rota siguiendo dos ejes, uno longitudinal y el otro anteroposterior; en el eje longitudinal, el estómago rota $90^{\circ}$ en el sentido de las manecillas del reloj, de manera que el lado izquierdo se orienta hacia delante y el lado derecho hacia atrás. Durante la rotación, la porción posterior original del estómago crece más rápido que la anterior, formándose las curvaturas mayor y menor. Dado que en esta etapa del desarrollo el estómago está unido a las paredes corporales posterior y anterior por los mesogastrios dorsal y ventral, respectivamente, se considera que la rotación del eje longitudinal tira del mesogastrio dorsal hacia la izquierda y ayuda a formar la bolsa omental o epiploica (fondo de saco peritoneal situado detrás del estómago). En un inicio, los extremos cefálico y caudal del estómago están situados en la línea media, pero al continuar el crecimiento, la porción caudal o pilórica se desplaza hacia la 

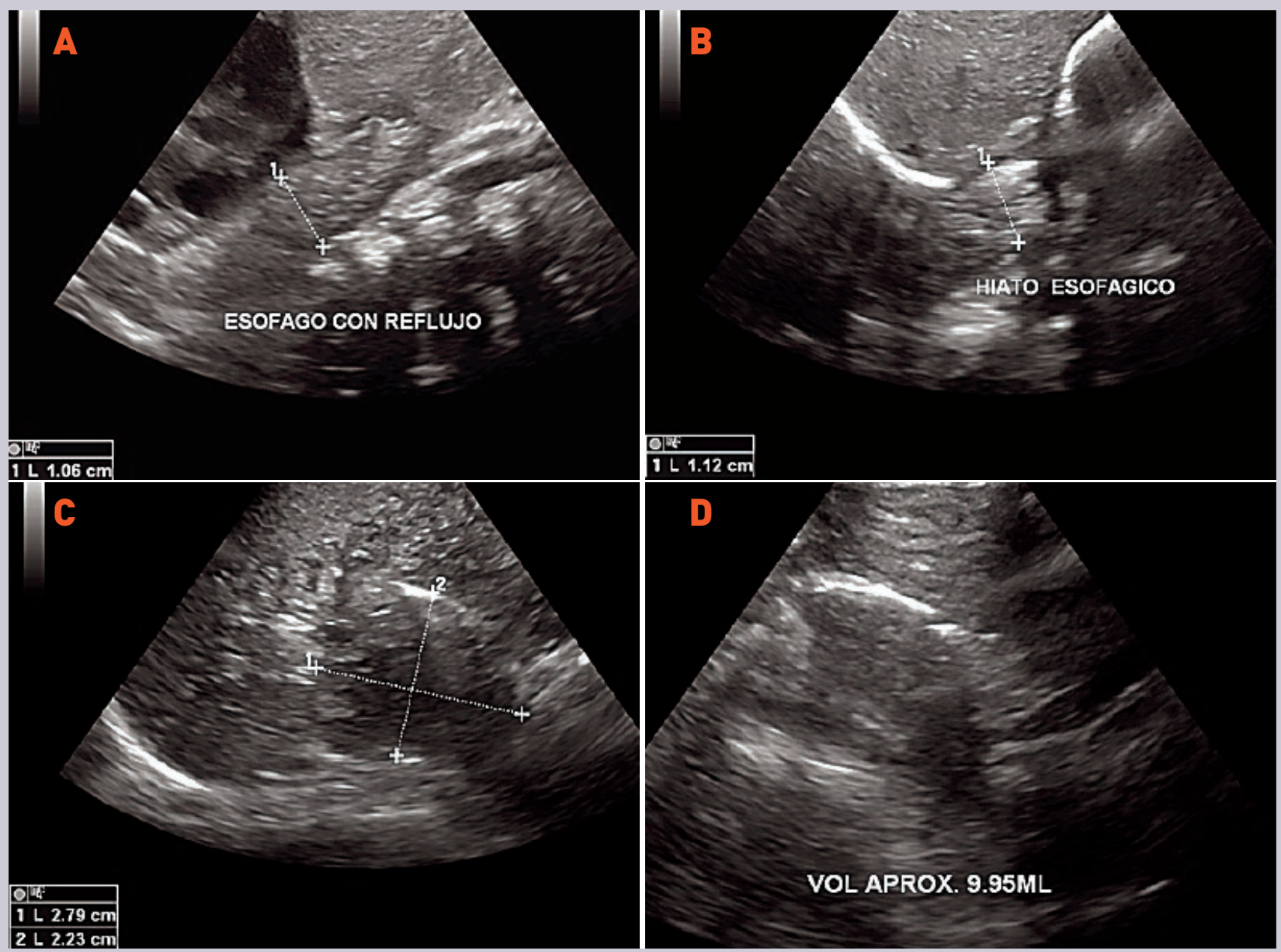

Figura 3. A: se observa paso del contenido gástrico desde el estómago hacia el esófago. B: el esófago se dilata por el contenido gástrico, con un diámetro de hasta $15 \mathrm{~mm}$. C: el estómago es redondeado y pequeño. D: estómago con un volumen aproximado de $10 \mathrm{ml}$.

derecha y arriba, y la porción cefálica o cardiaca se desplaza hacia la izquierda y abajo; de esta manera, el estómago adquiere su posición definitiva y el eje longitudinal desciende de izquierda a derecha. La curvatura mayor se orienta hacia abajo y la curvatura menor se orienta hacia arriba y a la derecha. Al mismo tiempo, el bazo surge en el mesogastrio dorsal por diferenciación mesenquimal. Por lo tanto, no son sorprendentes los hallazgos asociados de asplenia, porque tanto el bazo como el estómago son derivados del mesogastrio dorsal embrionario; sin embargo, en la paciente se observó el bazo por ecografía, y en el tránsito intestinal y la exploración de colon por enema se corroboraron la malrotación intestinal y el microcolon.

Las manifestaciones clínicas varían de un paciente a otro, y como la paciente estuvo en ayuno por hospitalización de 30 días, debido a enterocolitis necrosante y prematurez, los vómitos posprandiales mencionados se presentaron hasta después de ser dada de alta y fueron incrementando en número y cantidad hasta un total de ocho al día. 


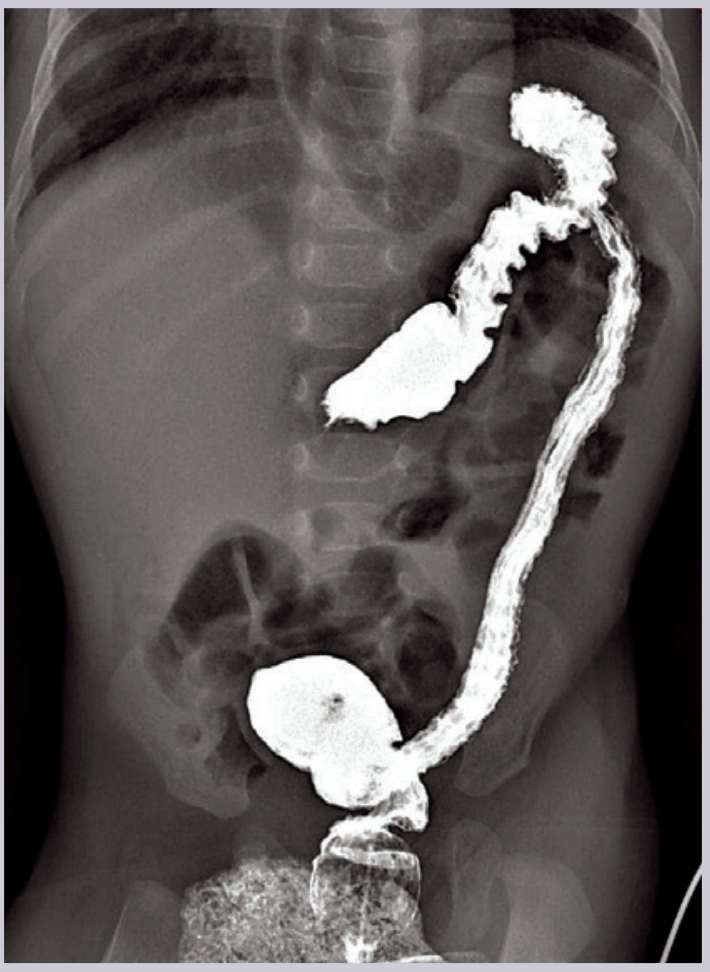

Figura 4. El colon se observa disminuido de calibre, tubular y con el ciego alto, sin aire intestinal del lado derecho y con el esófago dilatado.

Los clínicos pensaron que se trataba de una agenesia gástrica, pero el estómago nunca está completamente ausente, lo cual sería incompatible con la vida.

Como se refiere en la literatura, el diagnóstico se hizo con una SEGD. Se opacificaron el esófago y el estómago; sin embargo, el estómago regresaba el bario hacia el esófago en un ciclo continuo. El estómago se observó pequeño y de forma redonda, situado en la línea media y con el esfínter esofágico inferior permanentemente abierto.

La ecografía abdominal corroboró la capacidad gástrica disminuida $(10 \mathrm{ml})$ y el paso continuo del contenido gástrico hacia el esófago,

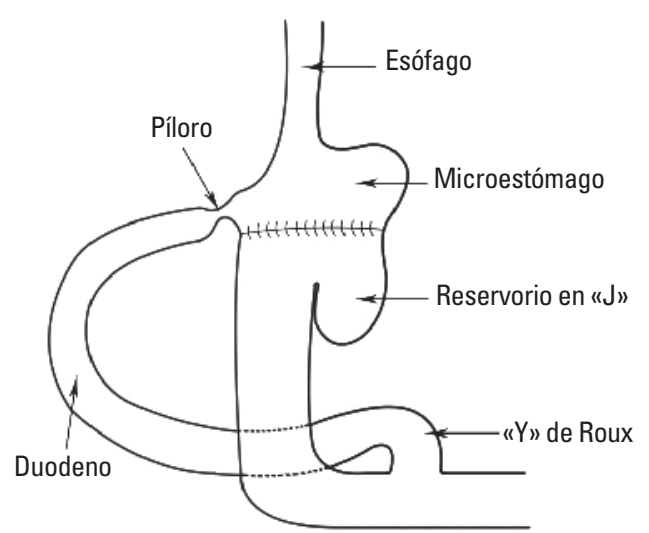

Figura 5. Bolsa yeyunal en "Y» de Roux de Hunt y Lawrence con reservorio en "J" (dibujo tomado de la hoja quirúrgica).

el cual permanecía lleno, dilatado y sin vaciamiento, siendo el primer caso reportado hasta el momento que corrobora los hallazgos de la SEGD.

El tratamiento de la microgastria congénita debe ser individualizado. Los pacientes con formas leves de microgastria pueden ser manejados conservadoramente, y aquellos con formas graves deben ser tratados quirúrgicamente. Los pacientes tratados en forma conservadora han sobrevivido con dieta frecuente en poca cantidad, pero su estómago no se agranda y tienen un crecimiento por debajo de lo normal, tanto en peso como en altura ${ }^{1,3}$. La gastrostomía y la gastroenterostomía han tenido un éxito limitado en el manejo de la microgastria congénita, porque no contemplan la necesidad de un reservorio como una medida para prevenir el $\mathrm{RGE}^{7}$. Hunt y Lawrence realizaron un procedimiento con el fin de restablecer la continuidad del tracto gastrointestinal después de la gastrectomía total por cáncer gástrico. 
La cirugía consiste en una bolsa para el reservorio de alimentos, que disminuye la necesidad de dieta frecuente y logra tener una alimentación balanceada ${ }^{3}$. En 1980, Neifeld utilizó la bolsa yeyunal en «Y» de Roux de Hunt y Lawrence para crear un reservorio gástrico en un paciente con microgastria congénita; la bolsa yeyunal aumentó la capacidad contenedora del estómago, facilitó el vaciamiento y previno la esofagitis por reflujo ${ }^{1,3-6,8,10,11}$. Con la bolsa yeyunal en «Y» de Roux de Hunt y Lawrence, la mayoría de los pacientes con microgastria congénita aumentan de peso y de altura, aunque estos permanecen de moderadamente a muy retrasados, y el resultado final en el desarrollo social e intelectual de los pacientes con microgastria dependerá de la presencia de anomalías asociadas. El retraso físico que se establece durante la infancia es difícil de revertir y continúa hasta la edad adulta en la mayoría de los pacientes con microgastria congénita.

En la paciente se decidió por el procedimiento de Hunt y Lawrence con el fin de ampliar el estómago con una bolsa yeyunal en «Y» de Roux y crear un reservorio gástrico en «J». A 3 semanas de la cirugía se suspendió la nutrición parenteral, se retiró la sonda orogástrica y desde su egreso la paciente ha tolerado la vía oral a razón de $60 \mathrm{ml}$ cada 2 horas, con un peso a su egreso de 3,620 g, y continúa asintomática hasta el día de hoy, con buen pronóstico para la vida. Seguirá siendo atendida por la consulta externa.

Probablemente la mejor alternativa para optimizar el crecimiento somático es llevar a cabo el procedimiento de la bolsa yeyunal en
«Y» de Roux de Hunt y Lawrence, en lugar de retrasar lo inevitable con medidas conservadoras que solo mantienen al niño con microgastria congénita en estado de desnutrición crónica.

\section{CONFLICTO DE INTERESES}

Los autores declaran que no hay conflicto de intereses en este estudio.

\section{FINANCIACIÓN}

Los autores declaran que no recibieron financiación para este estudio.

\section{BIBLIOGRAFÍA}

1. Hochberger O, Swoboda W. Congenital microgastria. A follow-up observation over six years. Pediat Radiol. 1974;2:207-8.

2. Gray SW, Skandalakis JE. The embriological basis for the treatment of congenital defects. En: Embryology for surgeons. Philadelphia: W.B. Saunders Company; 1972. p.105-6.

3. Kroes EJ, Festen C. Congenital microgastria: a case report and review of literature. Pediart Surg Int. 1998;13:416-8.

4. Sharma SC, Menon P. Congenital microgastria with esophageal stenosis and diaphragmatic hernia. Pediatric Surg Int. 2005;21:292-4.

5. Moulton SL, Bouvet M, Lynch FP. Congenital microgastria in a premature infant. J Pediatr Surg. 1994;29:1594-5.

6. Jones VS, Cohen RC. An eighteen-year follow-up after surgery for congenital microgastria - case report and review of literature. J Pediatr Surg. 2007;42:2957-60.

7. Kunisaki SM, Dakhoub A, Jarboe MD, Geiger JD. Gastric dissociation for the treatment of congenital microgastria with paraesophageal hiatal hernia. J Pediatr Surg. 2011;46:E1-E4.

8. Anderson KD, Guzzetta PC. Treatment of congenital microgastria and dumping syndrome. J Pediatr Surg. 1983;18:747-50.

9. Shackelford GD, McAlister WH, Brodeur AE, Ragsdale EF. Congenital microgastria. AJR Am J Roentgenol. 1973;118:72-6.

10. Filisetti C, Maestri L, Meroni M, Marinoni F, Riccipetitoni G. Severe dumping syndrome in a 6-years-old girl with congenital microgastria treated by Hunt-Lawrence pouch. Eur J Pediatr Surg Rep. 2017;5:e17-e20.

11. Zamakhshary M, Oda O, Alrabeeah A, Jadaan SA, Al-Namshan M, Crankson $S$, et al. A valid treatment option for isolated congenital microgastria. Ann Pediatr Surg. 2011;7:114-6.

12. Langman J. Porción caudal del intestino anterior. En: Embriología médica. 3. a ed. México: Interamericana;1976. p. 256-7. 